\title{
Usage of aspheric IOL design: the way forward
}

Mohtar Ibrahim

Department of Ophthalmology, School of Medical Sciences, Universiti Sains Malaysia, Kubang Kerian, Kelantan, Malaysia

In general, surgery induces structural changes to tissues. These changes are permanent. The minimum change is scarring, and the maximum is very much dependent on the complexity of the surgery. The more delicate the tissue or organ, the more care is needed to minimize the changes that result from surgical interventions.

The eye is a delicate and intricate organ. It is a known fact that no matter how minimal an intervention on the eye there is an effect in terms of its visual function. This is due to the fact that any surgical intervention induces physical 'defects' in the form of scars. It also induces other effects, such as inflammation, especially if the surgical intervention involves intraocular tissue manipulation. In addition, implantation of artificial intraocular lenses (IOLs) adds more consequences to these issues.

Cataract surgery is probably the most common intraocular surgery performed worldwide. As this surgery involves intervention of many ocular tissues, albeit 'minimal', it is impossible to restore the eye to its normal state. According to Christopher Kent, Senior Editor of Review of Ophthalmology, there are at least 25 ways to maximize cataract surgery outcomes that include pre-, intra-, and postoperative steps and measures. ${ }^{1}$ These suggestions include using a seven-variable IOL power calculation, an aspheric IOL, intraoperative aberrometry, and personalising the A-constant. It is also important that we 'individualise' every patient's precondition of the eye to predict the postoperative outcomes of cataract surgery.

In this modern and advanced era of cataract surgery, we cataract surgeons have to consider not only surgical techniques and IOL materials and design, we are also facing challenges in terms of increasing expectations on the part of patients for postoperative visual outcomes. Cataract surgery outcomes are no longer confined to improvement of distant and near visual acuity, but also other aspects of visual function, including contrast sensitivity and depth of focus. Improvement in contrast sensitivity is a function of 'restoring' the spherical aberration of the normal eye. ${ }^{2}$

Currently, there are many models of aspheric IOLs on the market, thanks to Bausch \& Lomb who introduced the first aspheric IOL model in 2004. ${ }^{3}$ These developments have spurred numerous studies around the globe. Using aspheric IOLs has nowadays become the gold standard in cataract management. 
It is heartening to note that the study conducted locally by Mae-Lynn et al. published in this issue of Malaysian Journal of Ophthalmology - has enlightened us on the outcomes of various IOLs that use aspheric principles. It is important to note, as I have stated earlier, that we have to cater to individual daily needs in managing every cataract patient, ensuring most of our pseudophakic patients are back to as near normal as possible.

\section{References}

1. Kent C. 25 Ways to Maximize Your Cataract Outcomes. Review of Ophthalmology. 2016 March 4. Available from: https://www.reviewofophthalmology.com/article/25-ways-to-maximize-your-cataract-outcomes.

2. Orlich C. Understanding spherical aberration. Eyeworld. Jan 2018. Available from: https://www. eyeworld.org/understanding-spherical-.

3. Samalonis LB. Aspheric IOLs: from Theory to Practice. Review of Ophthalmology. June 152005 . Available from: https://www.reviewofophthalmology.com/article/aspheric-iols-from-theory-to-practice. 